\title{
Neighborhood disadvantage across the transition from adolescence to adulthood and risk of metabolic syndrome
}

\author{
Chantel L. Martin ${ }^{\mathrm{a}, \mathrm{b}, *}$, Jennifer B. Kane ${ }^{\mathrm{b}, \mathrm{c}}$, Gandarvaka L. Miles ${ }^{\mathrm{a}}$, Allison E. Aiello ${ }^{\mathrm{a}, \mathrm{b}}$, \\ Kathleen Mullan Harris ${ }^{\mathrm{b}, \mathrm{d}}$

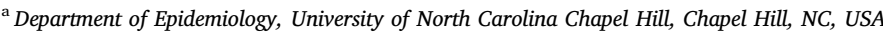 \\ ${ }^{\mathrm{b}}$ Carolina Population Center, University of North Carolina Chapel Hill, Chapel Hill, NC, USA \\ ${ }^{\mathrm{c}}$ Department of Sociology, University of California, Irvine, Irvine, CA, USA \\ ${ }^{\mathrm{d}}$ Department of Sociology, University of North Carolina Chapel Hill, Chapel Hill, NC, USA
}

\section{A R T I C L E I N F O}

\section{Keywords:}

Neighborhood disadvantage

Metabolic syndrome

Sensitive periods

Life course epidemiology

Population health

\begin{abstract}
A B S T R A C T
This study investigates the association between neighborhood disadvantage from adolescence to young adult hood and metabolic syndrome using a life course epidemiology framework. Data from the United States-based National Longitudinal Study of Adolescent to Adult Health $(\mathrm{n}=9500)$ and a structural equation modeling approach were used to test neighborhood disadvantage across adolescence, emerging adulthood, and young adulthood in relation to metabolic syndrome. Adolescent neighborhood disadvantage was directly associated with metabolic syndrome in young adulthood. Evidence supporting an indirect association between adolescent neighborhood disadvantage and adult metabolic syndrome was not supported. Efforts to improve cardiometabolic health may benefit from strategies earlier in life.
\end{abstract}

\section{Introduction}

Metabolic syndrome-clinically defined by the presence of central adiposity, dyslipidemia, hypertension, and insulin sensitivity (Grundy et al., 2005)—has been linked to increased risk of adverse cardiometabolic health, including cardiovascular disease and type 2 diabetes (Galassi et al., 2006; Laaksonen et al., 2002; Malik et al., 2004; McNeill et al., 2005). Cardiovascular disease is the leading cause of death in the United States (Heron, 2018) and, while risk factors can manifest as early as childhood, diagnosis generally occurs much later in life (Morrison et al., 2007). Therefore, metabolic syndrome is a reasonable outcome in studies of cardiometabolic risk in young adults, before permanent biological damage occurs, to help identify at-risk populations prior to the onset of cardiovascular disease. The prevalence of metabolic syndrome is relatively low during early life (3\% among children), but becomes increasingly prevalent in adulthood, with $23-44 \%$ of adults $\geq 19$ years of age impacted (Alexander et al., 2003; Beltrán-Sánchez et al., 2013; Friend et al., 2013). Given metabolic syndrome's rising prevalence with age, it is increasingly important to determine risk factors earlier in the life course that contribute to the development of metabolic syndrome for cardiovascular disease prevention.

Individual-level determinants of metabolic syndrome are well- established, including factors such as low socioeconomic status (Chichlowska et al., 2009; Gustafsson et al., 2011; Langenberg et al., 2006; Schooling et al., 2008; Yang et al., 2017), physical inactivity (He et al., 2014; Zhang et al., 2017), and poor diet quality (Lutsey et al., 2008; Rodriguez-Monforte et al., 2017). Within the last two decades, researchers have begun to acknowledge the importance of neighborhood-level conditions, beyond the previously mentioned individuallevel risk factors, in shaping cardiovascular health (Barber et al., 2016; Diez-Roux et al., 1997; Diez Roux et al., 2001, 2016; Diez Roux et al., 2002; Gebreab et al., 2017; Glass et al., 2006; Krishnan et al., 2010; Suglia et al., 2016). Individuals residing in disadvantaged neighborhoods experience limited food and physical activity environments, including limited availability and access to healthy food choices (Morland et al., 2002) and poor infrastructures for physical activity (GordonLarsen et al., 2006). Moreover, residing in disadvantaged neighborhoods is associated with increased exposure to chronic stressors, such as greater crime, discrimination, and despair (Ross and Mirowsky, 2001; Sampson et al., 1997). Taken together, determining the role of neighborhood-level disadvantage, independent of individual-level factors, in shaping the development of metabolic syndrome may be helpful in identifying targets for intervention to reduce the burden of cardiovascular disease.

Prior cross-sectional studies of neighborhood-level SES (i.e.

\footnotetext{
* Corresponding author. Carolina Population Center, 123 West Franklin St., Chapel Hill, NC 27516, USA

E-mail address: chantelmartin@unc.edu (C.L. Martin).
} 
disadvantage) and metabolic syndrome have consistently suggested that residing in more disadvantaged neighborhoods confer increased risk of metabolic syndrome (Chichlowska et al., 2008; Clark et al., 2013; Keita et al., 2014). However, such research designs preclude inference of the temporal effects of neighborhood disadvantage on metabolic syndrome and limits the ability to account for factors that select individuals into a given neighborhood (Diez Roux, 2004; Oakes, 2004). Therefore, it remains unclear whether contemporaneous associations between neighborhood-level disadvantage and metabolic syndrome are partially or entirely confounded by earlier life exposures.

Prospective studies of neighborhood disadvantage across the life course offer a unique opportunity to understand the mechanisms linking early life exposures to adult health. Life course epidemiology, which posits that social and biological factors may likely act independently, cumulatively, and interactively over the life course to impact adult health and disease (Kuh et al., 2003), provides a useful framework for conceptualizing these mechanisms. Residing in a disadvantaged neighborhood is correlated across life stages, such that individuals who experience neighborhood disadvantage in early life are more likely to experience adult neighborhood disadvantage (Gustafsson et al., 2013; Van Ham et al., 2014), with both likely influencing cardiometabolic health. Prior research suggests that residing in disadvantaged neighborhoods for longer time periods is associated with overweight and obesity (Do and Zheng, 2017; Lippert, 2016; Lippert et al., 2017; Sheehan et al., 2017), weight gain (Powell-Wiley et al., 2014), high blood pressure and hypertension (Lippert et al., 2017), and cardiovascular mortality (Xiao et al., 2018).

A focus of life course epidemiology has been identifying exposures during gestation, childhood, adolescence, and early adulthood that impact health outcomes later in life. Two theoretical models within the life course epidemiology framework may help to understand how neighborhood disadvantage in earlier life stages influence metabolic syndrome in adulthood: (1) the sensitive period model; and (2) chains of risk model. The sensitive period model describes a life stage when an exposure has a greater impact on disease risk than it would at other life stages (Kuh and Shlomo, 2004). To test the sensitive period life course model, control for subsequent life course exposures to neighborhood disadvantage is necessary. Under this life course model, neighborhood disadvantage during adolescence may serve as a sensitive period directly altering an individual's susceptibility to metabolic syndrome in adulthood by impacting their likelihood to develop unhealthy norms around diet and physical activity.

Second, a chains of risk model, a form of the accumulation of risk life course model, postulates that exposures are linked over the life course to influence later health (Kuh and Shlomo, 2004). Two possible chains of risk models exist: one whereby earlier exposure increases risk of later exposures while also independently impacting disease risk, and another whereby earlier exposures set off a chain of exposures that has no direct (independent) effect on disease risk except through the final link in the chain of exposures. Applied to the present study, it is plausible that living in a highly disadvantaged neighborhood early in life is associated with an increased risk of living in a highly disadvantaged neighborhood in adulthood, which in turn is associated with an increased risk of metabolic syndrome in adulthood. Early life exposure to a disadvantaged neighborhood may operate entirely through this pathway (and thus retain only an indirect effect on adulthood risk of metabolic syndrome) or may operate partially through this pathway (and thus retain an indirect and direct effect on adulthood risk of metabolic syndrome).

Longitudinal measures of neighborhood attributes are necessary for testing life course models and to more accurately assess long-term, dynamic exposures. Building upon previous research, this study applies a life course framework and structural equation modeling (SEM) approach to examine the sensitive period and chains of risk models to determine the association between neighborhood disadvantage during the transition from adolescence to adulthood and metabolic syndrome during adulthood. Using data from a nationally representative, longitudinal study of adolescents followed into adulthood, we explicitly tested whether: (1) the association between neighborhood disadvantage in adolescence and metabolic syndrome in adulthood remains after controlling for neighborhood disadvantage in subsequent life stages (sensitive period model); or (2) the association between neighborhood disadvantage in adolescence and metabolic syndrome in adulthood operates through subsequent neighborhood disadvantage (i.e. chains of risk model).

\section{Methods}

The National Longitudinal Study of Adolescent to Adult Health (Add Health) is an ongoing, nationally representative longitudinal study of adolescents in grades 7-12 during the 1994-1995 school year in the United States (Harris et al., 2013). Students were recruited from 132 middle and high schools. In 1994, in-school surveys were administered to 90,118 students selected from a stratified random sample of all high schools. A subsample of these students was randomly selected from the school rosters to participate in home-based interviews $(n=20,745)$. A second wave of in-home interviews were conducted among those in grades 8-12 in 1996 followed by a third wave of data collection in 2001-2002 during emerging adulthood (ages 18-26) and a fourth wave in 2008-2009 during young adulthood (ages 24-32) among Wave I participants who participated in the in-home survey.

Individuals included in our study were those who participated in Waves I, III, and IV ( $\mathrm{n}=13,034)$ without missing data on any component of the metabolic syndrome measure taken at Wave IV $(\mathrm{n}=11,422)$. We only included females who were not pregnant at the time of any of the three interviews $(n=10,762)$, as anthropometric and physiological markers do not compare among pregnant and non-pregnant women. Further, only US-born participants were included ( $n=10,077$ ) due to the established health differences by nativity status (Crosnoe, 2006; Harris et al., 2009; Hummer et al., 1999; Singh and Miller, 2004). Finally, participants with available Wave IV sampling weights to produce nationally-representative estimates were included resulting in a final analytic sample size of 9500 . We compared sociodemographic characteristics of those excluded from our analytic sample $(\mathrm{n}=7711)$ to those who responded across all three waves ( $n=13,034)$. Participants who responded across each of the three waves were more likely to be non-Hispanic white $(p=0.01)$, have a parent with more than a high school education $(\mathrm{p}<0.001)$, and have lived in their current residence since birth ( $p<0.001)$, but no difference was observed by parent age at baseline $(\mathrm{p}=0.58)$. Further, among the individuals who participated across all three waves $(n=13,034)$, we compared characteristics for those who were excluded due to missing metabolic syndrome components $(n=1612)$ and the participants who remained in the sample $(n=11,422)$. Similarly, we observed that those with all metabolic syndrome measures were more likely to be non-Hispanic white $(\mathrm{p}=0.001)$ and had slightly higher mean body mass index (BMI) at baseline $(\mathrm{p}=<0.01)$, but did not differ on educational attainment $(\mathrm{p}=0.37)$.

\subsection{Exposures}

Neighborhood characteristics were measured at the census-tract level using contextual data appended to Add Health (Harris, 2013). Information from the 1990 U.S. Census and the 2005-2009 U.S. American Community Survey were used to measure neighborhood disadvantage during adolescence (Wave I), emerging adulthood (Wave III), and young adulthood (Wave IV). Five census-tract level indicators were chosen a priori based on availability of measures in the 1990 and 2005-2009 instruments to reflect aspects of neighborhood income/ wealth, education, and household structure (Sampson et al., 1997). The five census-tract level indicators included: percent households with incomes below the federal poverty line; percent of households receiving public assistance; civilian unemployment rate; percent of persons 25 
Table 1

ATP III definition and modified ATP III definition (Add Health) for metabolic syndrome.

\begin{tabular}{|c|c|c|}
\hline Component & ATP III Definition & Modified ATP III Definition \\
\hline Central obesity & $\begin{array}{l}\text { High Waist Circumference: } \\
\text { Women: } \geq 88 \mathrm{~cm} \\
\text { Men: } \geq 102 \mathrm{~cm}\end{array}$ & ATP III Criteria \\
\hline High blood pressure & $\begin{array}{l}\text { Systolic/diastolic blood pressure: } \\
\geq 130 / 85 \mathrm{~mm} \mathrm{Hg} \text { or antihypertensive drug treatment }\end{array}$ & ATP III Criteria \\
\hline Reduced HDL-cholesterol & $\begin{array}{l}\text { HDL-cholesterol level: } \\
\text { Men: }<40 \mathrm{mg} / \mathrm{dL} \\
\text { Women: }<50 \mathrm{mg} / \mathrm{dL}\end{array}$ & $\begin{array}{l}\text { HDL-cholesterol level: } \\
\text { Men: lowest two deciles } \\
\text { Women: lowest decile }\end{array}$ \\
\hline Elevated triglycerides & $\begin{array}{l}\text { Triglyceride level: } \\
\quad \geq 150 \mathrm{mg} / \mathrm{dL}\end{array}$ & $\begin{array}{l}\text { Triglyceride levels: } \\
\text { Men: highest three deciles } \\
\text { Women: highest two deciles }\end{array}$ \\
\hline Insulin sensitivity & $\begin{array}{l}\text { Fasting glucose level: } \\
\quad \geq 110 \mathrm{mg} / \mathrm{dL}\end{array}$ & $\begin{array}{l}\text { HbA1c* level: } \\
\quad>5.6 \%\end{array}$ \\
\hline
\end{tabular}

years or older with no high school diploma or equivalency; and percent of female-headed households. To determine the final neighborhood disadvantage construct, we used factor analysis with Varimax orthogonal factor rotation and indicators were included based on the factor loadings, standardized regression coefficients, and measurement model fit.

\subsection{Outcome}

During young adulthood (Wave IV, ages 24-32), metabolic syndrome was defined according to the third Adult Treatment Panel (ATP III) guidelines (Grundy et al., 2005) and included the following five components: waist circumference, blood pressure, high density lipoprotein-cholesterol (HDL-c), triglycerides, and insulin resistance. Participants were classified as having metabolic syndrome if they met at least 3 of the 5 criteria. The ATP III definition was modified slightly to align with the available Add Health data (Table 1). First, the lab that provided the assayed specimens for lipids used two different assays for samples. After extensive data cleaning and quality control efforts, Add Health only released the rank-ordering (by deciles) as a more reliable measure than the absolute measures from conversion strategies. Therefore, we departed from the clinical cutoffs $(<50 \mathrm{mg} / \mathrm{dL}$ for women, $<40 \mathrm{mg} / \mathrm{dL}$ in men, or lipid lowering drug treatment) to classify reduced HDL-C as membership in the lowest category for women and lowest two deciles for men. This was based on evidence showing $11.9 \%$ of women and $31.4 \%$ of men have reduced HDL-C (Carroll et al., 2012). Similarly, the top three deciles of triglycerides for men and the top two deciles of triglycerides for women were used to define elevated triglycerides based on national estimates for men (29.6\%) and women (17.8\%) 20-39 years of age (Ervin, 2009). Second, insulin sensitivity was defined using pre-diabetic value of glycosylated hemoglobin (HbA1c) instead of the ATP III definition of fasting glucose $\geq 100 \mathrm{mg} / \mathrm{dL}$ or antidiabetic medication as participants were not required to fast before their interview. As a result, data contain a combination of both fasting and non-fasting glucose measures. Instead of combining the two measures, we used HbA1c, a measure of blood glucose in a person's body over 2-3 months prior to their interview. HbA1c provides a more stable measure of metabolic dysregulation than glucose, which varies widely by dietary intake prior to measurements. Other studies have also utilized the similar alternative definitions for measures of metabolic syndrome in Add Health (Gaydosh et al., 2018; Kane et al., 2017).

\subsection{Covariates}

Covariates included participants' baseline age (Wave I), sex, race/ ethnicity (Non-Hispanic white, Non-Hispanic black, Hispanic, other), parental educational attainment based on highest level achieved by mother and father (less than high school, high school or equivalent, some college, college degree or more), same residence since birth (yes/ no), and participant's baseline self-reported health using general question of health status (excellent, very good, good, fair, poor). Self-reported health was included as a covariate to account for potential residential selection issues due to childhood health status. Values were grouped to create a three-level variable - excellent/very good, good, fair/poor. Variables were defined as shown in Table 1.

\subsection{Statistical analysis}

We employed a structural equation modeling (SEM) approach to examine the pathways linking neighborhood disadvantage across the transition from adolescence to young adulthood on metabolic syndrome in young adulthood. Fig. 1 depicts a theoretical model of life course neighborhood disadvantage and metabolic syndrome, which was used to guide our analysis. SEM models include a measurement model (i.e. factor model) and structural model (i.e. regression) to estimate the direct and indirect effects. The findings from the factor analysis was tested in the measurement model (latent constructs for neighborhood disadvantage across each time period). The structural model estimated: (1) direct effect of adolescent neighborhood disadvantage on young adult metabolic syndrome, independent of all other pathways (sensitive periods model); and (2) indirect effect of adolescent neighborhood disadvantage on young adult metabolic syndrome, mediated by emerging adulthood neighborhood disadvantage and/or young adult neighborhood disadvantage (chains of risk model). We included sociodemographic and health-related factors as controls (age, sex, race/ ethnicity, parental education, living in same residence since birth, and self-reported health) to adjust for potential confounding.

All SEM analyses were conducted in MPlus version 8 (Muthén \& Muthén, Los Angelos, CA). We used a probit link function, theta parameterization, and weighted least squares estimator (WLSMV) to appropriately model the binary outcome, metabolic syndrome (yes versus no). Missing covariate data $(\mathrm{n}=56$ missing parent education, 21 missing race/ethnicity, 74 missing residence at birth, and 3 missing child self-reported health) were accounted for in MPlus using full information maximum likelihood estimation. Goodness-of-fit statistics for the final models were calculated using the root mean squared error of approximation (RMSEA) and comparative fit index (CFI). Models with a RMSEA $<0.05$, CFI $>0.90$, and SRMR $<0.08$ were considered to fit the data well (Bentler, 1990; Browne and Cudeck, 1993; Hu and Bentler, 1999).

\section{Results}

Weighted descriptive statistics, adjusted for clustering of the sample design, for selected adolescent and adult characteristics of Add Health participants included in this analysis are shown in Table 2 . The study sample had a mean age of 15 years, $50 \%$ were female, and $72 \%$ were 


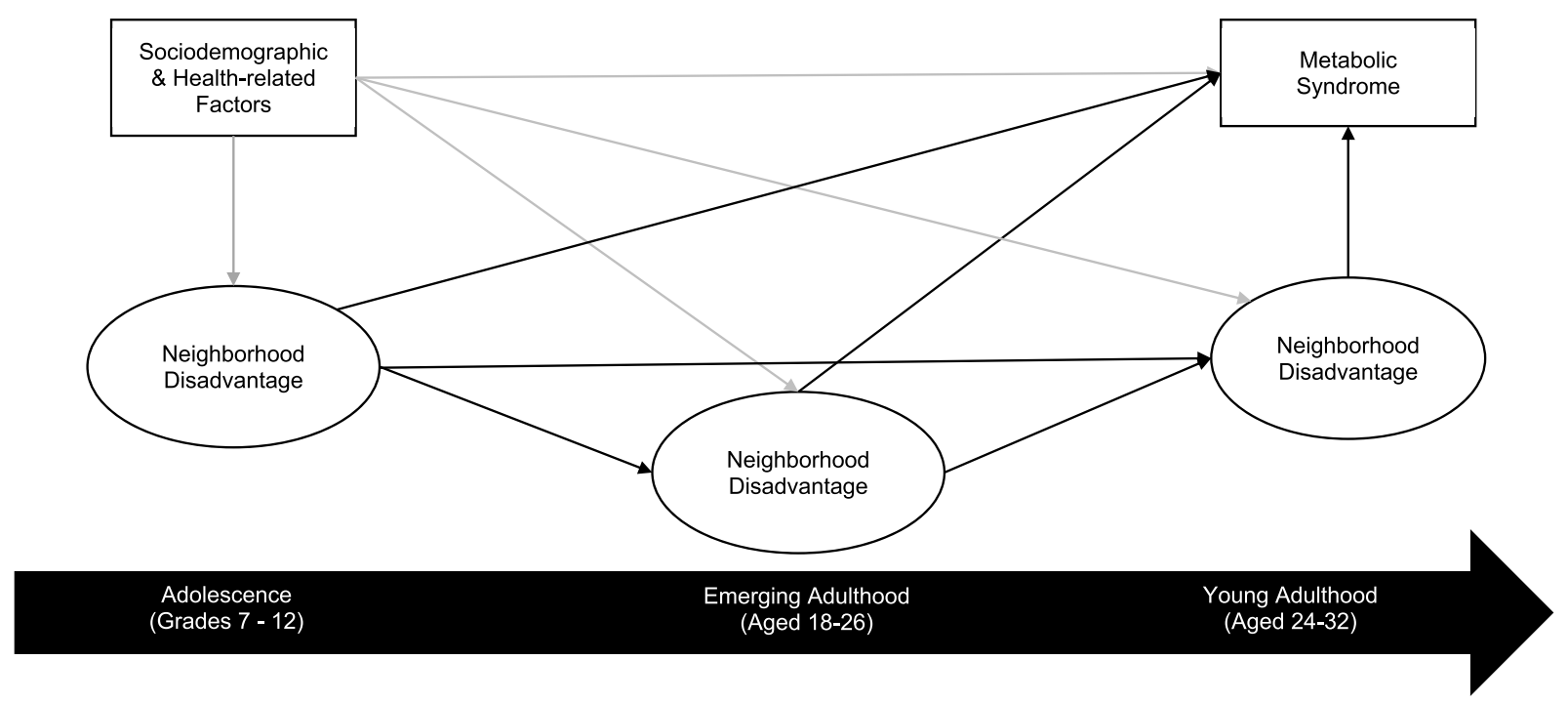

Fig. 1. Conceptual model of neighborhood disadvantage from adolescence to young adulthood and metabolic syndrome in young adulthood. Black lines represent paths estimated in the SEM. Gray lines reflect potential confounding pathways not directly estimated in the SEM.

Table 2

Descriptive statistics ${ }^{\mathrm{a}}$ of selected characteristics; National Longitudinal Study of Adolescent to Adult Health (Add Health) from 1994 to $2008(\mathrm{n}=9500)$.

\begin{tabular}{lll}
\hline & N (\%) & Mean (SE) \\
\hline Adolescent characteristics (Wave I) & & \\
Age in 1994 (years) & & \\
Sex & & \\
$\quad$ Female & $5092(50.1)$ \\
$\quad$ Male & $4408(50.0)$ & \\
Race/ethnicity & $5687(72.2)$ \\
$\quad$ Non-Hispanic white & $2007(14.7)$ \\
$\quad$ Non-Hispanic black & $1238(9.6)$ \\
$\quad$ Hispanic & $547(3.5)$ \\
$\quad$ Other & $1035(10.4)$ \\
Parental Education & $2443(27.4)$ \\
$\quad$ < High school & $5966(62.1)$ \\
$\quad$ High school or equivalent & & \\
More than high school & $2071(21.6)$ \\
Lived at same residence since birth & $7355(78.4)$ \\
$\quad$ Yes & \\
$\quad$ No & $6355(66.8)$ \\
Childhood self-reported health & $2426(25.9)$ \\
Excellent/Very good & $716(7.3)$ \\
$\quad$ Good & \\
$\quad$ Fair/Poor & $5041(51.9)$ \\
Metabolic syndrome (Wave IV) & $3985(43.4)$ \\
Central obesity (high waist circumference) & $2342(25.7)$ \\
Elevated blood pressure & $1519(16.6)$ \\
Elevated triglycerides & $3254(32.0)$ \\
Reduced LDL-cholesterol & $2407(24.8)$ \\
Elevated HbA1c & \\
Metabolic Syndrome &
\end{tabular}

${ }^{\mathrm{a}} \mathrm{N}$ 's are unweighted and means and percentages are weighted with probability sampling weights and standard errors adjusted for the cluster design.

non-Hispanic white. Sixty-two percent of participants had at least one parent with some college education or higher. Twenty-five percent of the study sample was classified with metabolic syndrome. Of the measured risk factors of metabolic syndrome, $52 \%$ had central obesity, $43 \%$ with elevated blood pressure, $26 \%$ with elevated triglycerides, $17 \%$ with reduced LDL-cholesterol, and $32 \%$ with elevated HbA1c.

Table 3 describes the prevalence of neighborhood disadvantage characteristics during adolescence, emerging adulthood, and young adulthood. Adolescents in our study sample resided in neighborhoods characterized by $12 \%$ of families below the federal poverty level, $9 \%$ of families receiving public assistance, $27 \%$ of households without an adult with a high school diploma, and 7\% female-headed households. During emerging and young adulthood, neighborhoods in which participants resided had similar presence of families living below poverty with approximately $11 \%$ at both time points. Also, during emerging and young adulthood, the neighborhood presence of families receiving public assistance and households without an adult with a high school diploma declined, while the percent of female-headed increased.

\subsection{Measurement models}

Unstandardized and standardized factor loadings for neighborhood disadvantage during adolescence, emerging adulthood, and young adulthood are presented in Supplemental Table 1. Across each time point, indicators of the latent variables reflecting neighborhood disadvantage were statistically significant at $\mathrm{p}<0.001$ and the measurement model fit was appropriate (RMSEA $=0.032$, CFI $=0.938$, SRMR $=0.044$ ). A general SEM diagram depicting neighborhood disadvantage in adolescence in relation to metabolic syndrome in young adulthood is provided in Supplemental Fig. 1. Neighborhood disadvantage in adolescence was positively associated with neighborhood disadvantage in both emerging and young adulthood and neighborhood disadvantage in emerging adulthood was also positively associated with neighborhood disadvantage in young adulthood, accounting for all other pathways (Supplemental Table 2).

Is adolescence a sensitive period when neighborhood disadvantage is associated with metabolic syndrome in adulthood (sensitive period model)?

The adjusted total, direct, and indirect effects of neighborhood disadvantage across the transition from adolescence to young adulthood on metabolic syndrome are summarized in Table 4. The SEM fit the data well (RMSEA $=0.020, \mathrm{CFI}=0.913$, SRMR $=0.065$ ). In our model, neighborhood disadvantage in adolescence was associated with metabolic syndrome. A positive total effect of neighborhood disadvantage in adolescence on metabolic syndrome in adulthood was observed. The direct effect of neighborhood disadvantage in adolescence to metabolic syndrome in young adulthood, independent of indirect neighborhood disadvantage pathways in emerging and young adulthood and potential confounding factors-adolescent age, parent education, race/ethnicity, sex, childhood self-reported health, and resident of neighborhood at birth-was also observed (direct effect: standardized coefficient $=0.057$, p-value $=0.024$ ).

Does neighborhood disadvantage in adolescence impact metabolic syndrome in young adulthood through exposure to neighborhood disadvantage in emerging and young adulthood (chains of risk model)? 
Table 3

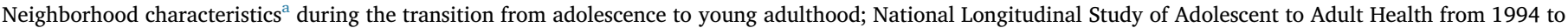
2008 ( $\mathrm{n}=9500)$.

\begin{tabular}{|c|c|c|c|}
\hline Neighborhood Variables & Adolescence (Wave I) \% (SE) & Emerging Adulthood (Wave III) \% (SE) & Young Adulthood (Wave IV) \% (SE) \\
\hline Families living below poverty level & $11.7(0.8)$ & $10.8(0.5)$ & $11.4(0.5)$ \\
\hline Families receiving public assistance & $8.6(0.5)$ & $3.6(0.2)$ & $2.6(0.1)$ \\
\hline Household with no adult having HS diploma & $26.8(0.9)$ & $20.0(0.7)$ & $15.6(0.5)$ \\
\hline Unemployed & $7.4(0.3)$ & $6.7(0.3)$ & $8.0(0.2)$ \\
\hline Female-headed households & $6.8(0.3)$ & $23.8(0.7)$ & $26.7(0.7)$ \\
\hline
\end{tabular}

a Statistics are weighted and adjusted for clustering.

Table 4

Standardized direct and indirect effects ${ }^{\mathrm{a}}$ of neighborhood disadvantage across the transition from adolescence to young adulthood and metabolic syndrome in young adulthood; Add Health from 1994 to 2008 (n = 9500).

\begin{tabular}{|c|c|c|}
\hline & \multicolumn{2}{|c|}{ Metabolic syndrome } \\
\hline & $\begin{array}{l}\text { Standardized } \\
\text { Estimate }\end{array}$ & p-value \\
\hline \multicolumn{3}{|l|}{ Adolescent Neighborhood Disadvantage } \\
\hline Total Effect & 0.076 & 0.001 \\
\hline Direct Effect & 0.057 & 0.024 \\
\hline Total Indirect Effect & 0.018 & 0.187 \\
\hline $\begin{array}{l}\text { Decomposition of Indirect Effects } \\
\text { via Emerging Adult Neighborhood } \\
\text { Disadvantage }\end{array}$ & 0.020 & 0.126 \\
\hline $\begin{array}{l}\text { via Young Adult Neighborhood } \\
\text { Disadvantage }\end{array}$ & -0.001 & 0.869 \\
\hline $\begin{array}{l}\text { via Emerging and Young Adult } \\
\text { Neighborhood Disadvantage }\end{array}$ & -0.001 & 0.869 \\
\hline \multicolumn{3}{|c|}{ Emerging Adult Neighborhood Disadvantage } \\
\hline Total Effect & 0.040 & 0.119 \\
\hline Direct Effect & 0.041 & 0.126 \\
\hline Total Indirect Effect & -0.001 & 0.869 \\
\hline Decomposition of Indirect Effects & & \\
\hline $\begin{array}{l}\text { Via Young Adult Neighborhood } \\
\text { Disadvantage }\end{array}$ & -0.001 & 0.869 \\
\hline \multicolumn{3}{|l|}{ Young Adult Neighborhood Disadvantage } \\
\hline Direct Effect & -0.004 & 0.869 \\
\hline
\end{tabular}

We did not find evidence of an indirect effect from neighborhood disadvantage in adolescence to metabolic syndrome in adulthood through neighborhood disadvantage in emerging and young adulthood (total indirect effect: standardized coefficient $=0.018, \mathrm{p}=0.19$ ). Furthermore, there was no evidence of direct effects between neighborhood disadvantage during emerging (total direct effect: standardized coefficient $=0.041, \mathrm{p}=0.13$ ) or young adulthood (total direct effect: standardized coefficient $=-0.004, p=0.87$ ) on metabolic syndrome, when adjusting for potential confounding factors and neighborhood disadvantage at earlier life stages.

\section{Discussion}

We tested two life course models to assess how neighborhood disadvantage across the life course influences metabolic syndrome in adulthood. First, we tested whether adolescence served as a sensitive period of development during which exposure to neighborhood disadvantage independently impacted metabolic syndrome in young adulthood. Our findings were in support of the sensitive period model, where neighborhood disadvantage in adolescence was associated with an increased risk of young adulthood metabolic syndrome, independent of all other hypothesized mediating pathways, including neighborhood disadvantage in emerging and young adulthood. Second, we tested whether a chains of risk model was supported across the life course. We did not find evidence of this life course model. Despite the finding that neighborhood disadvantage tracked across the transition from adolescence to young adulthood, the final "link" in the chains of neighborhood disadvantage was not associated with metabolic syndrome. Moreover, unlike previous research, neighborhood disadvantage in emerging adulthood and young adulthood was not associated with metabolic syndrome in young adulthood, after accounting for adolescent neighborhood disadvantage.

No previous study has assessed exposure to neighborhood disadvantage during early life (e.g. adolescence) as a sensitive period for development of metabolic syndrome; however, previous studies of life course neighborhood disadvantage in relation to weight status are suggestive of an association between early life exposure to neighborhood disadvantage and risk of obesity in adulthood (Harris et al., 2009; Kravitz-Wirtz, 2016). One study using data from the Panel Study on Income Dynamics to examine the timing of exposure to neighborhood disadvantage during childhood (e.g. early childhood [1-5 years], late childhood [6-11 years], and adolescence [12-17 years]) found that exposure to neighborhood disadvantage during adolescence was associated with greater odds of self-reported obesity at least once in early adulthood (18-30 years of age), independent of the childhood period (Kravitz-Wirtz, 2016).

Adolescence is an important stage of development characterized by increased desire for autonomy and greater exploration of one's neighborhood environment (Harris, 2010). During this time, adolescents begin making choices surrounding health-related behaviors (e.g. physical activity and dietary habits) that continue into adulthood (GordonLarsen et al., 2004; Biddle et al., 2010; Kane et al., 2017). At the same time, a large proportion of outside-the-home time spent by adolescents is in their residential neighborhoods, which provide physical space for social interactions (Leventhal et al., 2009), as well as provide contexts for food choices and physical activity. Neighborhood disadvantage has been associated with lower access to healthy food choices and recreational facilities (Gordon-Larsen et al., 2006; Morland et al., 2002). Collectively, exposure to neighborhood disadvantage during adolescence may begin to shape norms and attitudes around health behaviors that impact risk of metabolic syndrome. In addition to exposure to unhealthy behavioral practices, residing in disadvantaged neighborhoods exposes adolescents to stressful conditions that may bolster risk of poor cardiometabolic health in adulthood through increases in allostatic load and cardiometabolic-related physiologic dysfunction (McEwen, 2000). While beyond the scope of the current study, future research should explore such risk factors (e.g. health behaviors, mental health, socioeconomic position, stressors) that mediate the association between adolescent neighborhood disadvantage and young adulthood metabolic syndrome.

Interestingly, we did not find evidence of an indirect effect of neighborhood disadvantage in adolescence and metabolic syndrome in adulthood through neighborhood disadvantage in later life stages (i.e. young adulthood) or direct effects of neighborhood disadvantage in emerging and young adulthood on metabolic syndrome in adulthood when accounting for adolescent neighborhood disadvantage. The transition from adolescence to young adulthood introduces individuals to additional social environments (i.e. post-secondary education, work) that may diminish residential neighborhood effects in later life stages. 
The transitory nature of these early adult life stages may make residential locations less salient for health, given young adults likely view their current neighborhoods as non-permanent. Furthermore, our results offer a unique perspective in that neighborhood disadvantage in young adulthood may only serve as a proxy measure for earlier life disadvantage. Traditional methods that do not account for early life effects may result in bias due to missing historic confounding by antecedent early life environments. Indeed, when we examined the cross-sectional association between neighborhood disadvantage and metabolic syndrome during young adulthood without controlling for adolescent neighborhood disadvantage, a significant positive association was observed; however, this association diminished once we longitudinally account for earlier exposure to neighborhood disadvantage in adolescence as shown in our full model.

This study is not without limitations. First, self-reported weight and height were available at baseline to estimate BMI during adolescence, which might influence future neighborhood selection. We performed a sensitivity analysis to include adolescent BMI as a control measure influencing neighborhood disadvantage across the life course (i.e. adolescence, emerging adulthood, and young adulthood) and metabolic syndrome in adulthood and the findings of our SEM remained qualitatively unchanged. Second, although we controlled for important known factors that select individuals to reside in a given neighborhood (e.g., parent education, race/ethnicity, resident of neighborhood at birth), the potential for additional unmeasured confounding remains. Further, we did not include all possible pathways through which neighborhood disadvantage in adolescence might impact metabolic syndrome in adulthood as the focus of our paper was one specific pathway - neighborhood disadvantage in later life stages. However, future research will build on this work to further establish causality and explore additional pathways, such as health behaviors. Third, while adolescence is an important period of development during which neighborhood environment impacts metabolic syndrome development, earlier life stages, such as childhood or birth, may be most influential and adolescence serves only as a proxy. Unfortunately, we did not have data (i.e. census tract zip code) to assess neighborhood environment earlier in life; however, we were able to adjust for whether the participant was a resident of the neighborhood at birth using self-reported years lived at residence at baseline. In addition, a sensitivity analysis was conducted to adjust for self-reported birth weight as an early life proxy of health and socioeconomic status and our results did not change. Fourth, neighborhood boundaries were defined at the censustract level to compare our results to previous research. Census-tracts are subdivisions of counties; however, this may not capture the most salient residential environment, particularly among emerging and young adults as indicated in our findings. Lastly, the ATP III definition for metabolic syndrome was modified to align with our data. Specifically, decile ranks for cholesterol and triglycerides were used because absolute lipid levels were unavailable and hemoglobin A1C was used to represent insulin sensitivity as participants were not required to provide fasting blood samples. However, the decile ranks used in our analysis were comparable to the national prevalence of reduced HDL-cholesterol and elevated triglycerides during the time period of young adulthood and have been included in previous studies of metabolic syndrome in Add Health (Gaydosh et al., 2018; Lippert et al., 2017; Yang et al., 2017).
Despite these limitations, our study has important strengths. This is the first study to employ a life course framework to guide theoretical and statistical approaches for investigating neighborhood disadvantage during adolescence and metabolic syndrome in adulthood. This analysis was conducted in one of the largest, nationally representative, longitudinal studies in the United States with more than two decades of data collection. Because of Add Health's rich multi-level data across the life course, we were able to account for several factors (e.g. residence at birth, parental education) that may select adolescents into neighborhoods and also predict later life metabolic health. The findings of this study underscore the need for future research using multiple measures of neighborhood disadvantage to test other possible mechanisms across the life course that might mediate the effect of neighborhood disadvantage during adolescence on cardiometabolic health in adulthood.

In conclusion, the findings of this study are consistent with a sensitive period life course model - whereby the deleterious effects of neighborhood disadvantage in adolescence are associated with the risk of metabolic syndrome in adulthood, independent of later life experiences of neighborhood disadvantage. While exposure to neighborhood disadvantage in adolescence was associated with metabolic syndrome in adulthood, no association between neighborhood disadvantage in emerging adulthood or young adulthood was observed when accounting for neighborhood disadvantage in adolescence. Furthermore, we did not find evidence that neighborhood disadvantage during adolescence was operating through experiences of neighborhood disadvantage in later life to impact metabolic syndrome (chains of risk model), further supporting our findings of a sensitive period model. This study provides the most stringent test to date of the association between neighborhood disadvantage and metabolic syndrome and pinpoints the life stage in which these exposures are likely to be more influential for an individual's risk of developing metabolic syndrome in adulthood. Policy and practice decisions based on cross-sectional evidence provided to-date would have invested in concurrent neighborhoods and likely found little to no impact. Based on this new, longitudinal evidence, investments made earlier in the life course-during adolescence and maybe before-may be more effective in reducing the burden of adverse cardiometabolic health.

\section{Declaration of interests}

None.

\section{Acknowledgments}

This research uses data from the National Longitudinal Study of Adolescent to Adult Health (Add Health), a program project directed by Kathleen Mullan Harris and designed by J. Richard Udry, Peter S. Bearman, and Kathleen Mullan Harris at the University of North Carolina at Chapel Hill. The project was funded by grant P01HD31921 from the Eunice Kennedy Shriver National Institute of Child Health and Human Development, with cooperative funding from 23 other federal agencies and foundations. The research was supported by the National Institute on Minority Health and Health Disparities (K99MD012808), Eunice Kennedy Shriver National Institute of Child Health and Human Development (T32HD007186), and National Institute of Environmental Health Sciences (T32ES007018). 


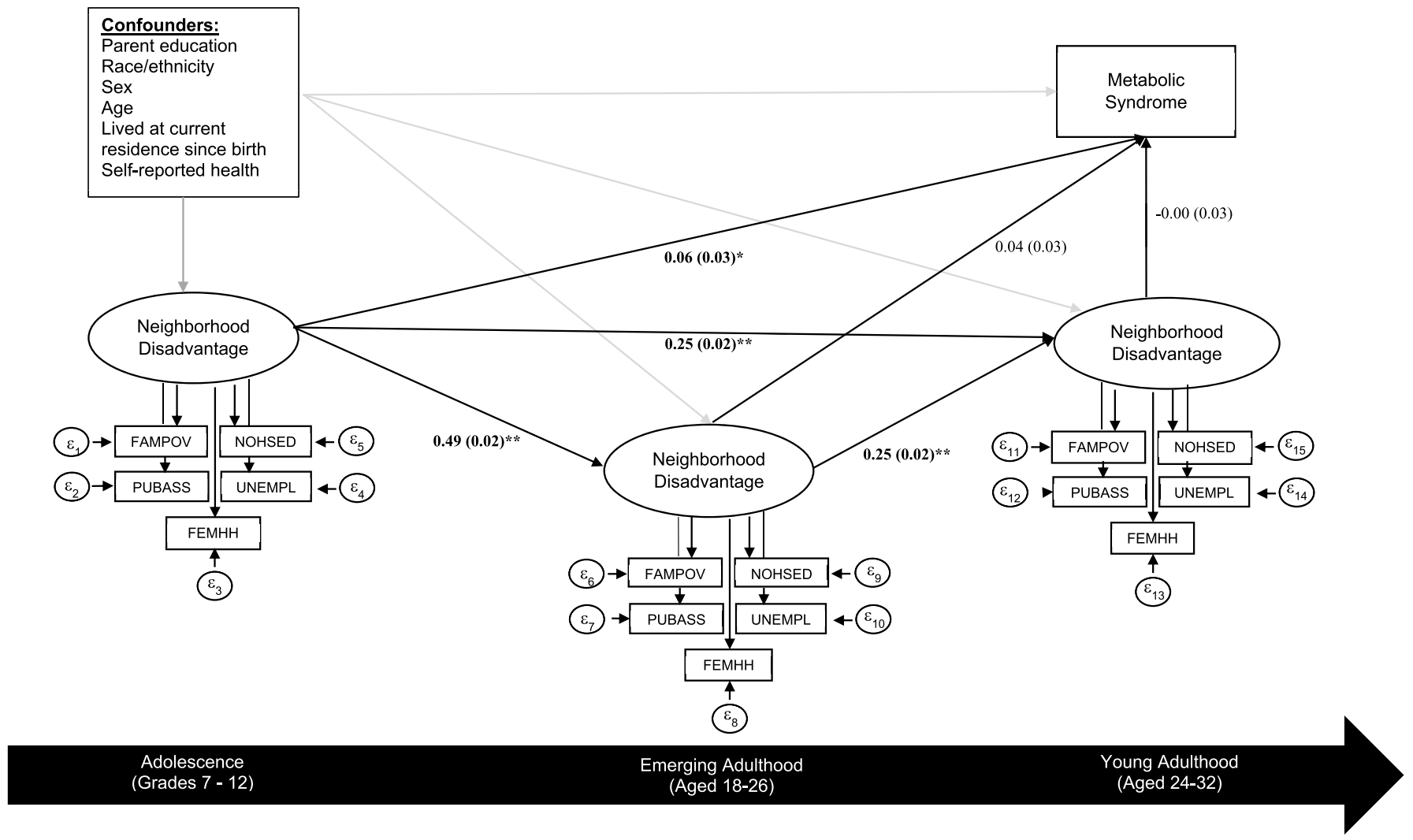

Supplemental Fig. 1. Diagram of SEM for standardized (standard errors) direct and indirect effects of neighborhood disadvantage in adolescence, emerging adulthood, and young adulthood on adult metabolic syndrome, Add Health. Solid black lines represent direct and indirect effects; gray lines represent potential confounding pathways. ${ }^{*} P<0.05 ; * * P<0.001$ (RMSEA $=0.020$; CFI $=0.913$; SRMR $=0.065$ ).

\section{Appendix A. Supplementary data}

Supplementary data to this article can be found online at https://doi.org/10.1016/j.healthplace.2019.03.002.

\section{References}

Alexander, C.M., Landsman, P.B., Teutsch, S.M., Haffner, S.M., 2003. NCEP-defined metabolic syndrome, diabetes, and prevalence of coronary heart disease among NHANES III participants age 50 Years and older. Diabetes 52 (5), 1210-1214.

Barber, Sharrelle, Hickson, Demarc A., Wang, Xu, Sims, Mario, Nelson, Cheryl, Diez-Roux, Ana V., 2016. Neighborhood disadvantage, poor social conditions, and cardiovascular disease incidence among African American adults in the Jackson heart study. Am. J. Public Health 106 (12), 2219-2226.

Beltrán-Sánchez, Hiram, Harhay, Michael O., Harhay, Meera M., McElligott, Sean, 2013. Prevalence and trends of metabolic syndrome in the adult U.S. Population, 19992010. J. Am. Coll. Cardiol. 62 (8), 697-703.

Bentler, P.M., 1990. Comparative fit indexes in structural models. Psychol. Bull. 107, 238-246.

Biddle Stuart, J.H., Pearson, Natalie, Ross Gemma, M., Braithwaite, Rock, 2010. Tracking of sedentary behaviours of young people: a systematic review. Prev. Med. 51 (5), 345-351.

Browne, M.W., Cudeck, R., 1993. In: Bollen, K.A., Long, J.S. (Eds.), Alternative Ways of Assessing Model Fit.

Carroll, Margaret D., Kit, Brian K., Lacher, David A., 2012. Total and high-density lipoprotein cholesterol in adults: national health and nutrition examination survey, 20092010. NCHS Data Brief (92), 1-8.

Chichlowska, K.L., Rose, K.M., Diez-Roux, Ana V., Golden, Sherita H., Mcneill, Annie M., Heiss, Gerardo, 2009. Life course socioeconomic conditions and metabolic syndrome in adults: the atherosclerosis risk in communities (ARIC) study. North 19 (12), 916-929.

Chichlowska, Kristal L., Kathryn, M. Rose, Diez-Roux, Ana V., Golden, Sherita H., McNeill, Annie M., Heiss, Gerardo, 2008. Individual and neighborhood socioeconomic status characteristics and prevalence of metabolic syndrome: the atherosclerosis risk in communities (ARIC) study. Psychosom. Med. 70 (9), 986-992.

Clark, Cheryl R., Ommerborn, Mark J., De Marc A, Hickson, Kya N, Grooms, Mario, Sims,
Taylor, Herman A., Albert, Michelle A., 2013. Neighborhood disadvantage, neighborhood safety and cardiometabolic risk factors in african Americans: biosocial associations in the Jackson heart study. PLoS One 8 (5).

Crosnoe, Robert, 2006. Health and the education of children from racial/ethnic minority and immigrant families. J. Health Soc. Behav. 47 (1), 77-93.

Diez-Roux, A.V., Nieto, F.J., Muntaner, C., Tyroler, H.A., Comstock, G.W., Shahar, E., Cooper, L.S., Watson, R.L., Szklo, M., 1997. Neighborhood environments and coronary heart disease: a multilevel analysis. Am. J. Epidemiol. 146 (1), 48-63.

Diez Roux, A.V., Merkin, S.S., Arnett, D., Chambless, L., Massing, M., Nieto, F.J., Sorlie, P., Szklo, M., Tyroler, H.A., Watson, R.L., 2001. Neighborhood of residence and incidence of coronary heart disease. N. Engl. J. Med. 345 (2), 99-106.

Diez Roux, Ana V., 2004. Estimating neighborhood health effects: the challenges of causal inference in a complex world. Soc. Sci. Med. 58 (10) 1953-50.

Diez Roux, Ana V., Jacobs, David R., Kiefe, Catarina I., 2002. Neighborhood characteristics and components of the insulin resistance syndrome in young adults: the coronary artery risk development in young adults (CARDIA) study. Diabetes Care 25 (11), 1976-1982.

Diez Roux, Ana V., Mujahid, Mahasin S., Hirsch, Jana A., Moore, Kari, Moore, Latetia V., 2016. The impact of neighborhoods on CV risk. Global Heart 11 (3), 353-363.

Do, D. Phuong, Zheng, Cheng, 2017. A marginal structural modeling strategy investigating short and long-term exposure to neighborhood poverty on BMI among U.S. Black and white adults. Health Place 46 (May), 201-209.

Ervin, R. Bethene, 2009. Prevalence of metabolic syndrome among adults 20 Years of age and over, by sex, age, race and ethnicity, and body mass index: United States, 20032006. Natl. Health Stat. Rep. (13), 1-7.

Friend, Amanda, Craig, Leone, Turner, Steve, 2013. The prevalence of metabolic syndrome in children: a systematic review of the literature. Metab. Syndrome Relat. Disord. 11 (2), 71-80.

Galassi, Andrea, Reynolds, Kristi, Jiang, He, 2006. Metabolic syndrome and risk of cardiovascular disease: a meta-analysis. Am. J. Med. 119 (10), 812-819.

Gaydosh, Lauren, Schorpp, Kristen M., Chen, Edith, Miller, Gregory E., Harris, Kathleen Mullan, 2018. College completion predicts lower depression but higher metabolic 
syndrome among disadvantaged minorities in young adulthood. Proc. Natl. Acad. Sci. Unit. States Am. 115 (1), 109-114.

Gebreab, Samson Y., DeMarc, A. Hickson, Mario, Sims, Wyatt, Sharon B., Davis, Sharon K., Correa, Adolfo, Diez-Roux, Ana V., 2017. Neighborhood social and physical environments and type 2 diabetes mellitus in african Americans: the Jackson heart study. Health Place 43, 128-137.

Glass, Thomas A., Rasmussen, Meghan D., Schwartz, Brian S., 2006. Neighborhoods and obesity in older adults: the baltimore memory study. Am. J. Prev. Med. 31 (6), $455-463$.

Gordon-Larsen, Penny, Nelson, Melissa C., Popkin, Barry M., 2004. Longitudinal physical activity and sedentary behavior trends: adolescence to adulthood. Am. J. Prev. Med. 27 (4), 277-283.

Gordon-Larsen, Penny, Nelson, Melissa C., Page, Phil, Popkin, Barry M., 2006. Inequality in the built environment underlies key health Disparities in physical activity and obesity. Pediatrics 117 (2), 417-424.

Grundy, Scott M., Cleeman, James I., Daniels, Stephen R., Donato, Karen A., Eckel, Robert H., Franklin, Barry A., Gordon, David J., Krauss, Ronald M., Savage, Peter J., Jr Smith, Sidney C., Spertus, John A., Costa, Fernando, 2005. Diagnosis and management of the metabolic syndrome: an American heart association/national heart, lung, and blood Institute scientific statement. Circulation 112 (17), 2735-2752.

Gustafsson, Per E., Mats, Persson, Anne, Hammarström, 2011. Life course origins of the metabolic syndrome in middle-aged women and men: the role of socioeconomic status and metabolic risk factors in adolescence and early adulthood. Ann. Epidemiol. 21 (2), 103-110.

Gustafsson, Per E., San Sebastian, Miguel, Janlert, Urban, Theorell, Töres, Westerlund, Hugo, Hammarström, Anne, 2013. Residential selection across the life course: adolescent contextual and individual determinants of neighborhood disadvantage in midadulthood. PLoS One 8 (11).

Van Ham, Maarten, Lina Hedman, Manley, David, Coulter, Rory, Östh, John, 2014. Intergenerational transmission of neighbourhood poverty: an analysis of neighbourhood histories of individuals. Trans. Inst. Br. Geogr. 39 (3), 402-417.

Harris, Kathleen Mullan, 2010. An integrative approach to health. Demography 47 (1), $1-22$.

Harris, Kathleen Mullan, 2013. The Add Health Study: Design and Accomplishments. Carolina Population Center, University of North Carolina at Chapel Hill.

Harris, Kathleen Mullan, Halpern, Carolyn Tucker, Hussey, Jon, Whitsel, Eric A., KilleyaJones, Ley, Tabor, Joyce, Elder, Glen, Hewitt, John, Shanahan, Michael, Williams, Redford, Siegler, Ilene, Smolen, Andrew, 2013. Social, behavioral, and genetic lin kages from adolescence into adulthood. Am. J. Public Health 103 (S1), S25-S32.

Harris, Kathleen Mullan, Perreira, Krista M., Lee, Dohoon, 2009. Obesity in the transition to adulthood: predictions across race/ethnicity, immigrant generation, and sex. Arch. Pediatr. Adolesc. Med. 163 (11), 1022-1028.

He, Dan, Xi, Bo, Xue, Jian, Huai, Pengcheng, Zhang, Min, Li, Jun, 2014. Association between leisure time physical activity and metabolic syndrome: a meta-analysis of prospective cohort studies. Endocrine 46 (2), 231-240.

Heron, Melonie, 2018. Deaths. Leading causes for 2016. Natl. Vital Stat. Rep. 67 (6), $1-76$.

Hu, Li Tze, Bentler, Peter M., 1999. Cutoff criteria for fit indexes in covariance structure analysis: conventional criteria versus new alternatives. Struct. Equ. Model. 6 (1), $1-55$.

Hummer, Robert A., Rogers, Richard G., Nam, Charles B., LeClere, Felicia B., 1999. Race/ ethnicity, nativity, and US adult mortality. Soc. Sci. Q. 136-153.

Kane, Jennifer B., Harris, Kathleen Mullan, Morgan, S. Philip, Guilkey, David K., 2017. Pathways of health and human capital from adolescence into young adulthood. Soc. Forces 96 (3), 949-976.

Keita, Akilah Dulin, Judd, Suzanne E., Howard, Virginia J., Carson, April P., Ard, Jamy D., Fernandez, Jose R., 2014. Associations of neighborhood area level deprivation with the metabolic syndrome and inflammation among middle- and older- age adults. BMC Public Health 14 (1), 1319.

Kravitz-Wirtz, Nicole, 2016. Temporal effects of child and adolescent exposure to neighborhood disadvantage on black/white Disparities in young adult obesity. J. Adolesc. Health 58 (5), 551-557.

Krishnan, Supriya, Cozier, Yvette C., Rosenberg, Lynn, Palmer, Julie R., 2010. Socioeconomic status and incidence of type 2 diabetes: results from the black women's health study. Am. J. Epidemiol. 171 (5), 564-570.

Kuh, D., Ben-Shlomo, Y., Lynch, J., Hallqvist, J., Power, C., 2003. Life course epidemiology (glossary). J. Epidemiol. Community Health 57 (10), 778-783.

Kuh, Diana, Shlomo, Yoav Ben, 2004. A Life Course Approach to Chronic Disease Epidemiology. Oxford University Press.

Laaksonen, David E., Lakka, Hanna-Maaria, Niskanen, Leo K., Kaplan, George A., Salonen, Jukka T., Lakka, Timo A., 2002. Metabolic syndrome and development of diabetes mellitus: application and validation of recently suggested definitions of the metabolic syndrome in a prospective cohort study. Am. J. Epidemiol. 156 (11), 1070-1077. Langenberg Claudia, Kuh, Diana, Michael, E, Wadsworth, J., Brunner, Eric, Hardy, Rebecca, 2006. Social circumstances and education: life course origins of social inequalities in metabolic risk in a prospective national birth cohort. Am. J. Public Health 96 (12), 2216-2221.

Leventhal, Tama, Dupéré, Véronique, Brooks-Gunn, Jeanne, 2009. Neighborhood influences on adolescent development. Handb. Adolesc. Psychol. 2, 411-443.

Lippert, Adam M., 2016. Stuck in unhealthy places. J. Health Soc. Behav. 57 (1), 1-21.

Lippert, Adam M., Evans, Clare Rosenfeld, Razak, Fahad, Subramanian, S.V., 2017. Associations of continuity and change in early neighborhood poverty with adult cardiometabolic biomarkers in the United States: results from the national longitudinal study of adolescent to adult health, 1995-2008. Am. J. Epidemiol. 185 (9), $765-776$.

Lutsey, Pamela L., Steffen, Lyn M., Stevens, June, 2008. Dietary intake and the development of the metabolic syndrome: the atherosclerosis risk in communities study. Circulation 117 (6), 754-761.

Malik, S., Wong, N.D., Franklin, S.S., Kamath, T.V., L'Italien, G.J., Pio, J.R., Williams, G.R., 2004. Impact of the metabolic syndrome on mortality from coronary heart disease, cardiovascular disease, and all causes in United States adults 1. Circulation 110 (1524-4539), 1245-1250 (Electronic).

McEwen, Bruce S., 2000. Allostasis and allostatic load: implications for neuropsychopharmacology. Neuropsychopharmacology 22 (2), 108-124.

McNeill, Ann, Marie, Rosamond, Wayne D., Girman, Cynthia J., Hill Golden, Sherita, Schmidt, Maria I., East, Honey E., Ballantyne, Christie M., Heiss, Gerardo, 2005. The metabolic syndrome and 11-year risk of incident cardiovascular disease in the atherosclerosis risk in communities study. Diabetes Care 28 (2), 385-390.

Morland, Kimberly, Wing, Steve, Roux, Ana Diez, Poole, Charles, 2002. Neighborhood characteristics associated with the location of food stores and food service places. Am. J. Prev. Med. 22 (1), 23-29.

Morrison, J.A., Friedman, L.A., Gray-McGuire, C., 2007. Metabolic syndrome in childhood predicts adult cardiovascular disease 25 Years later: the princeton lipid research clinics follow-up study. Pediatrics 120 (2), 340-345.

Oakes, J. Michael, 2004. The (Mis)Estimation of neighbor- hood effects: causal inference for a practicable so- cial epidemiology. Soc. Sci. Med. 58 (10), 1929-1952.

Powell-Wiley, Tiffany M., Ayers, Colby, Agyemang, Priscilla, Leonard, Tammy, Berrigan, David, Ballard-Barbash, Rachel, Lian, Min, Das, Sandeep R., Hoehner, Christine M., 2014. Neighborhood-level socioeconomic deprivation predicts weight gain in a multiethnic population: longitudinal data from the dallas heart study. Prev. Med. 66, 22-27.

Rodriguez-Monforte, Miriam, Sanchez, Emilia, Barrio, Francisco, Costa, Bernardo, FloresMateo, Gemma, 2017. Metabolic syndrome and dietary patterns: a systematic review and meta-analysis of observational studies. Eur. J. Nutr. 56 (3), 925-947.

Ross, Catherine E., Mirowsky, John, 2001. Neighborhood disadvantage, disorder, and health. J. Health Soc. Behav. 42 (3), 258-276.

Sampson, R.J., Raudenbush, S.W., Earls, F., 1997. Neighborhoods and violent crime: a multilevel study of collective efficacy. Science (New York, N.Y.) 277 (5328), 918-924.

Schooling, C.M., Jiang, C.Q., Lam, T.H., Zhang, W.S., Cheng, K.K., Leung, G.M., 2008. Life-course origins of social inequalities in metabolic risk in the population of a developing country. Am. J. Epidemiol. 167 (4), 419-428.

Sheehan, Connor M., Cantu, Phillip A., Powers, Daniel A., Margerison-Zilko, Claire E., Cubbin, Catherine, 2017. Long-term neighborhood poverty trajectories and obesity in a sample of California mothers. Health Place 46 (March), 49-57.

Singh, Gopal K., Miller, Barry A., 2004. Health, life expectancy, and mortality patterns among immigrant populations in the United States. Can. J. Public Health 95 (3), 14-21.

Suglia, Shakira F., Shelton, Rachel C., Hsiao, Amber, Claire Wang, Y., Rundle, Andrew, Link, Bruce G., 2016. Why the neighborhood social environment is critical in obesity prevention. J. Urban Health 93 (1), 206-212.

Xiao, Qian, Berrigan, David, Tiffany, M., Powell-Wiley, Matthews, Charles E., 2018. Tenyear change in neighborhood socioeconomic deprivation and rates of total, cardiovascular disease, and cancer mortality in older US adults. Am. J. Epidemiol. 187 (12), 2642-2650.

Yang, Yang Claire, Gerken, Karen, Schorpp, Kristen, Boen, Courtney, Kathleen, Mullan Harris, 2017. Early-life socioeconomic status and adult physiological functioning: a life course examination of biosocial mechanisms. Biodemogr. Soc. Biol. 63 (2), 87-103.

Zhang, Dongdong, Liu, Xuejiao, Liu, Yu, Sun, Xizhuo, Wang, Bingyuan, Ren, Yongcheng, Zhao, Yang, Zhou, Junmei, Han, Chengyi, Yin, Lei, Zhao, Jingzhi, Shi, Yuanyuan, Zhang, Ming, Hu, Dongsheng, 2017. Leisure-time physical activity and incident metabolic syndrome: a systematic review and dose-response meta-analysis of cohort studies. Metabolism 75, 36-44. 